\title{
Detecting and Tracking Community Dynamics in Evolutionary Networks
}

\author{
Zhengzhang Chen ${ }^{* \dagger}$, Kevin A. Wilson*‡, Ye Jin*†, William Hendrix ${ }^{* \dagger}$ and Nagiza F. Samatova* ${ }^{*} \S$ \\ * Department of Computer Science \\ North Carolina State University, Raleigh, NC, USA \\ ${ }^{\dagger}$ Computer Science and Mathematics Division \\ Oak Ridge National Laboratory, Oak Ridge, TN, USA \\ ${ }^{\ddagger}$ Research Computing Division, RTI International \\ Research Triangle Park, NC, USA \\ ${ }^{\S}$ Corresponding author: samatovan@ornl.gov
}

\begin{abstract}
Community structure or clustering is ubiquitous in many evolutionary networks including social networks, biological networks and financial market networks. Detecting and tracking community deviations in evolutionary networks can uncover important and interesting behaviors that are latent if we ignore the dynamic information. In biological networks, for example, a small variation in a gene community may indicate an event, such as gene fusion, gene fission, or gene decay. In contrast to the previous work on detecting communities in static graphs or tracking conserved communities in time-varying graphs, this paper first introduces the concept of community dynamics, and then shows that the baseline approach by enumerating all communities in each graph and comparing all pairs of communities between consecutive graphs is infeasible and impractical. We propose an efficient method for detecting and tracking community dynamics in evolutionary networks by introducing graph representatives and community representatives to avoid generating redundant communities and limit the search space. We measure the performance of the representative-based algorithm by comparison to the baseline algorithm on synthetic networks, and our experiments show that our algorithm achieves a runtime speedup of 11-46. The method has also been applied to two real-world evolutionary networks including Food Web and Enron Email. Significant and informative community dynamics have been detected in both cases.
\end{abstract}

Keywords-community detection; evolutionary analysis; social networks; community dynamics;

\section{INTRODUCTION}

Networks of dynamic systems can be highly clustered, or form community structures [18]. A community, defined as a collection of individual objects that interact unusually frequently, is a very common substructure in many networks [7], including social networks, metabolic and protein interaction networks, financial market networks, and even climate networks. In social networks, a community is a real social grouping sharing the same interests or background [7]. In biological networks, a community might represent a set of proteins that perform a distinct function together. Communities in financial market networks might denote groups of investors that own the same stocks, and communities in climate networks might indicate regions with a similar climate.
Many algorithms have been developed for detecting community structures in static graphs. Girvan and Newman [7] proposed a community discovery algorithm based on the iterative removal of edges with high "betweenness" scores. To reduce the computational cost of the betweennessbased algorithm, Clauset et al. [6] proposed a modularitybased algorithm. In contrast, Palla and Derenyi [12] did not focus on detecting separate communities, but on finding overlapping communities. Defining communities as maximal cliques, Schmidt et al. [14] proposed a parallel, scalable, and memory-efficient algorithm for their enumeration.

In addition, some work has been done on detecting conserved or stable communities in evolutionary networks. Hopcroft and Khan [9] proposed a method which utilizes a "nature community" to track stable clusters over time. A framework for identifying communities in dynamic social networks, proposed by Chayang and Berger-Wolf [17], makes explicit use of temporal changes. Using the Clique Percolation Method to locate communities, Palla et al. [13] defined auto-correlation and stationarity to characterize a community. They also introduced six basic community events, which will be used in our paper to define the community dynamics. But instead of focusing on the stationarity of the communities, our goal is to detect and track community dynamics. From an application perspective, Steinhaeuser $e t$ al. [16] provided a method to identify climate regions by detecting communities in time-varying climate networks.

However, communities in real networks change over time. In contrast to the previous work on tracking conserved communities, the evolution of structure within social networks has been addressed in [10]. Kumar et al. presented a model of network growth to capture singletons, isolated communities, and a giant component. Backstrom et al. [1] also considered the structural changes of social networks, but instead of detecting isolated communities, they focused on the structural features that influence the group growth for a period of time. In this paper, we not only consider grown communities, but also detect all the other types of community dynamics, including shrunken communities, merged communities, split communities, born communities, 
and vanished communities (see Definition 6).

Being able to detect all types of small community deviations can help us understand and exploit these networks more effectively. For example, in biological networks, a small variation in a gene community may represent an event, such as gene fusion [15], gene fission [15], gene gain [5], gene decay [11], or gene duplication [19], that would change the properties of the gene products (e.g., proteins) and consequently affect the phenotype of the organism. Interesting community deviation patterns in Food Web and social networks can be seen in Section IV-B.

Our approach follows from the need to address the following four issues:

- How do we define community dynamics, and how many types of community dynamics are possible in evolutionary networks? Community dynamics would reveal latent behaviors of the network, as opposed to conserved communities or communities in a single snapshot. For example, is there any community in snapshot $t$ that splits into smaller communities or merges with others in snapshot $k$ ? Does any community in snapshot $t$ disappear in snapshot $k$, or does any new community appear in snapshot $k$ ? Do the sizes of the communities change over time?

- Most real networks are dynamic and characterized by overlapping communities [12]. Detecting community dynamics from networks characterized by overlapping communities is more challenging than discovering separated communities in static networks.

- How do we detect and track community dynamics across multiple graphs? As we mentioned earlier, realworld networks change over time, requiring us to adopt evolutionary analysis techniques to detect and track these community dynamics.

- Since there may be hundreds or even thousands of communities in each real-world network, our algorithm needs to be able to scale to large graphs.

A naïve solution to these problems is to first enumerate all communities in each graph, and then compare all pairs of communities between two consecutive graphs. This twostage approach is infeasible and impractical because of the possible exponential number of communities and huge search space. For example, if we consider a maximal clique as a community, a graph can have up to $3^{\frac{n}{3}}$ communities, where $n$ is the number of vertices, and the possible pairs of communities between two consecutive graphs could be in the order of $3^{\frac{2 n}{3}}$. Meanwhile, there are many redundant communities (e.g., graph-dependent communities (see Definition 3)) in each graph, and it does not make much sense to compare pairs of communities that contain no common members or few members.

Therefore, in this paper, we propose an efficient algorithm based on the proposed notion of graph representatives and community representatives. Graph representatives can help us reduce the expensive computational cost caused by enumerating communities, which are modeled as maximal cliques in our paper. Community representatives can be exploited to identify the community dynamics.

The contributions of the paper are:

1) We prove that only six types of dynamic communities are possible in dynamic simple undirected graphs;

2) We develop an efficient algorithm for detecting and tracking community dynamics based on the introduced concept of graph representatives and community representatives; and

3) We evaluate our method on real datasets to confirm its applicability in practice.

\section{Problem Statement}

Table I

SYMBOL TABLE

\begin{tabular}{ll} 
Symbol & Description \\
\hline$G_{t}$ & A simple undirected labeled graph \\
$C_{t}^{i}$ & The graph sequence \\
$R e p\left(G_{t}\right)$ & The community of index $i$ in graph $G_{t}$ \\
$C_{t}^{i} \rightarrow C_{t+1}^{j}$ & The representative node set of graph $G_{t}$ \\
$T$ & $C_{t}^{i}$ is a predecessor of $C_{t+1}^{j}$ or $C_{t+1}^{j}$ is a successor \\
of $C_{t}^{i}$ & The number of timestamps in the sequence \\
$V\left(C_{t}^{i}\right)$ & The node set of community $C_{t}^{i}$ \\
$S V\left(G_{t}\right)$ & Common nodes between graphs $G_{t}$ and $G_{t+1}$ \\
$|C|$ & The size of community $C$ \\
$|N C|$ & The number of communities in a graph \\
$|V(C)|$ & The number of vertices in community $C$ \\
$v_{j}$ & A vertex $v_{j}$ in a graph \\
$C G_{t}$ & The list of communities in graph $G_{t}$ \\
$V C_{t}^{v_{j}}$ & The list of communities that contain node $v_{j}$ at \\
$N C_{t}^{v_{j}}$ & snapshot $t$ \\
$C h e c k e d\left(G_{t}\right)$ & The number of communities contain node $v_{j}$ at \\
$\emptyset$ & The list of nodes in $G_{t}$ that have been checked.
\end{tabular}

In this paper, the ultimate goal is to detect and track community dynamics in evolutionary networks, and our algorithm is based on graph representatives and community representatives. Thus, the following terms and problems need to be addressed. The symbols used throughout the paper are listed in Table I.

Problem 1 (Detecting and Tracking Community dynamics): Given a time-varying graph sequence $\mathcal{G}=\left\{G_{1}, G_{2}, G_{3}, \ldots\right\}$, detect and track the community dynamics, including grown communities, shrunken communities, merged communities, split communities, born communities, and vanished communities (see Definition 6).

Definition 1 (Community): Communities are the maximal cliques in the graph.

There is no formal definition for the community structure in a network [7]. The simplest and the most conservative definition of a community is a clique, a set of vertices that 
are adjacent to one another. Another definition used by Newman [7] is a dense subgraph, or a group of vertices within which the connections are denser than between different groups. Because our goal is to detect community dynamics, we prefer the more specific definition (clique) rather than the fuzzy one (dense subgraph). More importantly, from an application perspective, we may lose important changing trend information by using the dense subgraph definition. For example, consider protein functional modules (biological communities) in protein-protein interaction networks. Two overlapping communities in one organism may merge in another organism to affect a particular phenotype (e.g. hydrogen/ethanol production, high temperature resistance, nitrogen fixation, or aerobic respiration). If we consider a dense subgraph as a community, then the merging event for two overlapping communities will become hard or even impossible to detect. Also, our target applications necessitate a simpler and more stringent definition of a community-a clique.

This form of abstraction has been found beneficial in various application domains, such as those in biology. Specifically, such an abstraction was found to be less sensitive to noise inherent to biological networks and better at uncovering biologically relevant protein complexes [8]. Moreover, in the context of community dynamics, a small perturbation to the community structure, such as a single edge or vertex deletion, may indicate important events, such as gene fusion [15], gene fission [15], gene gain [5], gene decay [11], or gene duplication [19]. While useful in some application domains, clique-based modeling of a community might be inadequate in other application domains such as those in social networks.

Definition 2 (Community size): Community size, $|C|$, is the number of vertices in the community, so $|C|=|V(C)|$.

Definition 3 (Graph representative): Representatives of graph $G_{t}$ are the nodes that also appear in $G_{t-1}, G_{t+1}$, or both, thus $\operatorname{Rep}\left(G_{t}\right)=\left\{v_{i} \mid v_{i} \in G_{t} \bigcap G_{j}\right.$, where $|j-t|=$ $1\}$.

Nodes that only appear in one graph are called graphdependent nodes/vertices. If a community only contains graph-dependent vertices, then it can be considered as a "graph-dependent" community, which isn't a community dynamic we try to detect. Thus, using graph representatives as seeds, we do not need to enumerate all communities in the graphs, but only the communities containing the representatives. This technique can potentially reduce computational time (see Section III for details).

Definition 4 (Community predecessor and successor): If one community $C_{t}^{i}$ at snapshot $t$ is the subset or superset of the community $C_{t+1}^{j}$ at snapshot $t+1$, then the community $C_{t}^{i}$ is a predecessor of $C_{t+1}^{j}$ and $C_{t+1}^{j}$ is a successor of $C_{t}^{i}$. And the community relationship is defined by $C_{t}^{i} \rightarrow C_{t+1}^{j}$.

Definition 5 (Community representative): Community representative of $C_{t}^{i}$ is a node in $C_{t}^{i}$ that has the minimum number of appearances in other communities of the same graph. If there is more than one node that satisfies this condition, we choose one at random.

The rationale for our definition of a community representative follows from the observation that the community $C_{t}^{i}$ can be represented by a node that only appears in community $C_{t}^{i}$. However, since the communities in our networks may be highly overlapping, we cannot guarantee that such a node exists, so we look for a node in $C_{t}^{i}$ that has the minimum number of appearances in other communities to use as its representative. In this way, we limit the nodes that belong to more than one community from being a community representative, which helps to establish the relationships between the communities (see Section III for details).

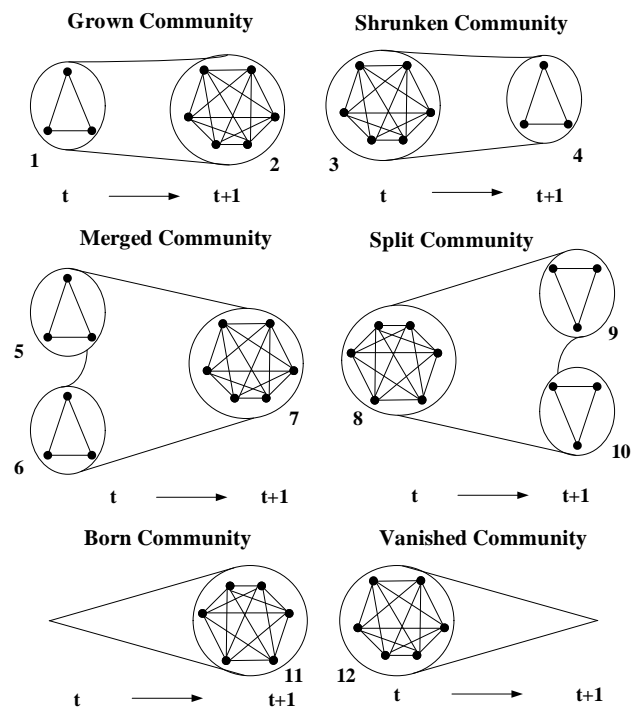

Figure 1. Possible types of community dynamics in evolutionary networks.

Definition 6 (Community dynamics): In contrast to [13], which focuses on the stability/stationarity of the communities, our goal is to detect and track community dynamics. As there are six basic events that may occur to a community [13], we can define six possible types of community dynamics in evolutionary networks (see Fig. 1).

1) Grown community

In real-world networks, some "large" communities, like community 2 , are grown from previous "smaller" communities by adding some new members.

2) Shrunken community

On the other hand, the shrunken communities, like community 4 , are the communities shrunk from previous "bigger" communities by losing some members.

3) Merged community

In addition, often two or more "small" communities at snapshot $t$ join together to be one merged community, like community 7 , at snapshot $t+1$.

4) Split community 
Meanwhile, a split community, like community 8, at snapshot $t$ are broken up into more than one different communities at snapshot $t+1$.

5) Born community

What's more, some "new" communities, like community 11 , would appear in some snapshots.

6) Vanished community

Finally, some "old" communities, like community 12 , would disappear.

\section{REPRESENTATIVE-BASED AlgORITHM FOR DETECTING AND TRACKING COMMUNITY DYNAMICS}

In this section, we first describe some necessary lemmas and theories. Then, based on the dynamic community decision rules described in Section III-B, we illustrate how to detect dynamic communities in SectionIII-C.

\section{A. Lemmas and Theorems}

Lemma 1: If community $C_{t}^{i}$ has more than one predecessor, the sizes of its predecessors are either all larger than $\left|C_{t}^{i}\right|$ or all smaller than $\left|C_{t}^{i}\right|$.

Proof: Suppose otherwise, that, let $C_{t-1}^{1}, C_{t-1}^{2}, \ldots$, $C_{t-1}^{n}$ (where $n \geq 2$ ) be all predecessors of $C_{t}^{i}$, and there is one predecessor $C_{t-1}^{j}$ with $\left|C_{t-1}^{j}\right| \leq\left|C_{t}^{i}\right|$ and another predecessor $C_{t-1}^{k}\left|C_{t-1}^{k}\right| \geq\left|C_{t}^{i}\right|$ for some $1 \leq j, k \leq n$, $j \neq k$. From Definition 4 and the sizes of the three communities, we know that $C_{t-1}^{j} \subseteq C_{t}^{i}$ and $C_{t}^{i} \subseteq C_{t-1}^{k}$, so $C_{t-1}^{j} \subseteq C_{t-1}^{k}$. However, $C_{t-1}^{j}$ and $C_{t-1}^{k}$, where $j \neq k$, are two different cliques in the same graph, so $C_{t-1}^{j} \subset C_{t-1}^{k}$. In addition, $C_{t-1}^{j}$ and $C_{t-1}^{k}$ are both maximal cliques in the same graph, so $C_{t-1}^{j} \subset C_{t-1}^{k}$ contradicts the definition of a maximal clique. Therefore, it is impossible to have one predecessor with larger size than the community, and another predecessor with smaller size.

Similarly, we can prove that if a community $C_{t}^{i}$ has more than one successor, the sizes of its successors are either all larger than $\left|C_{t}^{i}\right|$ or all smaller than $\left|C_{t}^{i}\right|$.

Theorem 1: Let $G_{t}$ and $G_{t+1}$ both be simple, undirected graphs, where communities are defined as maximal cliques. If $G_{t+1}$ is the perturbed graph formed by either adding edges/nodes to or removing edges/nodes from the baseline graph $G_{t}$, then there are only six possible types of community dynamics between $G_{t}$ and $G_{t+1}$ : grown communities, shrunken communities, merged communities, split communities, born communities, and vanished communities, as defined in Definition 6.

Based on Lemma 1, it is straightforward to prove Theorem 1. But for the sake of completeness, we present our proof here.

Proof: Assume that $C_{t}^{1}, C_{t}^{2}, \ldots, C_{t}^{m}$ are all communities in $G_{t}$ and that $V_{t}^{1}, V_{t}^{2}, \ldots, V_{t}^{m}$ are the node sets of the communities, respectively. Also assume that $C_{t+1}^{1}, C_{t+1}^{2}, \ldots, C_{t}^{n}$ are all communities in $G_{t+1}$ and that
$V_{t+1}^{1}, V_{t+1}^{2}, \ldots, V_{t+1}^{n}$ are the node sets of the communities, respectively. To determine the type of a specific community, we only need to compare the node sets of communities in $G_{t+1}$ with the node sets of communities in $G_{t}$. If $V_{t+1}^{j}=V_{t}^{i}$, where $1 \leq i \leq m$ and $1 \leq j \leq n$, then community $C_{t+1}^{j}$ contains exactly those nodes in community $C_{t}^{i}$, which means that $C_{t+1}^{j}$ is a conserved community and not a community dynamic. However, for communities $C_{t}^{i}$ and $C_{t+1}^{j}$ that do not have a "match" in $G_{t+1}$ or $G_{t}$, respectively, then there are overall seven possibilities.

1) For a specific $j$ (where $1 \leq j \leq n$ ), there is at least one $i$ (where $1 \leq i \leq m$ ) that satisfies $V_{t+1}^{j} \subset V_{t}^{i}$. Then, by Definition 4 , community $C_{t+1}^{j}$ has at least one predecessor, including $C_{t}^{i}$, with larger size than $C_{t+1}^{j}$. Let $I=\left\{i \mid V_{t+1}^{j} \subset V_{t}^{i}\right\}$. There are two nonexclusive sub-cases here:

a) For $\ell \in I$, if there is some $k$ (where $1 \leq k \leq n$ ) other than $j$ that satisfies $V_{t+1}^{k} \subset V_{t}^{\ell}$, then $C_{t}^{\ell}$ has more than one smaller-size successor $\left(C_{t+1}^{j}\right.$ and $\left.C_{t+1}^{k}\right)$. By Lemma $1, C_{t}^{\ell}$ cannot have a successor with larger size than $C_{t}^{j}$. Thus, $C_{t}^{\ell}$ is a split community, and $C_{t+1}^{j}$ is one of its products.

b) For $\ell \in I$, if there is no $k$ (where $1 \leq k \leq n$ ) other than $j$ that satisfies $V_{t+1}^{k} \subset V_{t}^{\ell}$, then $C_{t}^{\ell}$ has only one smaller-size successor $C_{t+1}^{j}$, and $C_{t+1}^{j}$ has at least one predecessor, including $C_{t}^{\ell}$, with larger size. Also, by Lemma 1, we know that $C_{t+1}^{j}$ cannot have a predecessor with smaller size than $C_{t+1}^{j}$. Thus, $C_{t+1}^{j}$ is a shrunken community.

2) For a specific $j$ (where $1 \leq j \leq n$ ), there is only one $i$ (where $1 \leq i \leq m$ ) that satisfies $V_{t+1}^{j} \supset V_{t}^{i}$. Then, community $C_{t+1}^{j}$ has one predecessor $C_{t}^{i}$ with smaller size than $C_{t+1}^{j}$. Additionally, by Lemma 1, we know that $C_{t+1}^{j}$ cannot have a predecessor with larger size than $C_{t+1}^{j}$. Thus, $C_{t+1}^{j}$ is a grown community.

3) For a specific $j$ (where $1 \leq j \leq n$ ), there is more than one $i$ (where $1 \leq i \leq m$ ) that satisfies $V_{t+1}^{j} \supset$ $V_{t}^{i}$. Then, $C_{t+1}^{j}$ has more than one predecessor with smaller size. Also, by Lemma $1, C_{t+1}^{j}$ cannot have a predecessor with larger size than $C_{t+1}^{j}$. Thus, $C_{t+1}^{j}$ is a merged community.

4) For a specific $j$ (where $1 \leq j \leq n$ ), there is no $i$ (where $1 \leq i \leq m$ ) that satisfies $V_{t+1}^{j} \supset V_{t}^{i}$ or $V_{t+1}^{j} \subset V_{t}^{i}$, which means that community $C_{t+1}^{j}$ has no predecessor. Thus, $C_{t+1}^{j}$ is a born community.

5) For a specific $i$ (where $1 \leq i \leq m$ ), there is at least one $j$ (where $1 \leq j \leq n$ ) that satisfies $V_{t+1}^{j} \subset V_{t}^{i}$. Let $J=\left\{j \mid V_{t+1}^{j} \subset V_{t}^{i}\right\}$. Then, for each $k \in J$, there is at least one $i$ (where $1 \leq i \leq m$ ) that satisfies $V_{t+1}^{k} \subset V_{t}^{i}$, which is case 1 . Thus, this case can be converted to case 1 .

6) For a specific $i$ (where $1 \leq i \leq m$ ), there is at least 
one $j$ (where $1 \leq j \leq n$ ) that satisfies $V_{t+1}^{j} \supset V_{t}^{i}$. Let $J=\left\{j \mid V_{t+1}^{j} \supset V_{t}^{i}\right\}$. Then, for each $k \in J$, there is at least one $i$ (where $1 \leq i \leq m$ ) that satisfies $V_{t+1}^{k} \supset V_{t}^{i}$, which is case 2 or 3 . Thus, this case can be converted to case 2 or 3 .

7) For a specific $i$ (where $1 \leq i \leq m$ ), there is no $j$ (where $1 \leq j \leq n$ ) that satisfies $V_{t+1}^{j} \supset V_{t}^{i}$ or $V_{t+1}^{j} \subset$ $V_{t}^{i}$, which means that community $C_{t}^{i}$ has no successor. Thus, $C_{t}^{i}$ is a vanished community.

Since all relationships between $V_{t+1}^{j}$ (where $1 \leq j \leq n$ ) and $V_{t}^{i}$ (where $1 \leq i \leq m$ ) have been covered, there are only six possible different types of community dynamics.

\section{B. Decision Rules for Community Dynamics}

The following decision rules for community dynamics hold based on Definition 6 and Theorem 1:

1) If community $C_{t}^{i}$ has only one predecessor $C_{t-1}^{j}$ :

a) If the size of the predecessor is smaller than $\left|C_{t}^{i}\right|$, then $C_{t}^{i}$ is a grown community.

b) If the size of the predecessor is larger than $\left|C_{t}^{i}\right|$ and $C_{t}^{i}$ is the only successor of $C_{t-1}^{j}$, then $C_{t}^{i}$ is a shrunken community.

c) If the size of the predecessor is larger than $\left|C_{t}^{i}\right|$ and $C_{t}^{i}$ is not the only successor of $C_{t-1}^{j}$, then $C_{t}^{i}$ is a product of the split community $C_{t-1}^{j}$.

2) If community $C_{t}^{i}$ has more than one predecessor:

a) If the sizes of the predecessors are all smaller than $\left|C_{t}^{i}\right|$, then $C_{t}^{i}$ is a merged community.

b) If the sizes of the predecessors are all larger than $\left|C_{t}^{i}\right|$ and $C_{t}^{i}$ is the only successor of one of its predecessors, then $C_{t}^{i}$ is a shrunken community.

c) If the sizes of the predecessors are all larger than $\left|C_{t}^{i}\right|$ and $C_{t}^{i}$ is not the only successor of one of its predecessors, then that community is a split community and $C_{t}^{i}$ is one of its products.

3) If community $C_{t}^{i}$ has no predecessor, then $C_{t}^{i}$ is a born community.

4) If community $C_{t}^{i}$ has no successor, then $C_{t}^{i}$ is a vanished community.

\section{Algorithm Description}

\section{Non-representative-based Method:}

To detect and track the community dynamics without using graph or community representatives, we first enumerate all communities in each graph, and then we compare all pairs of communities belonging to consecutive timestamps. For example, to find the successors of community $A$ in Fig. 2, we need to compare community $A$ with communities $D, E, F$, and $K$ at snapshot $t+1$; that is, we compare community $A$ with all communities at snapshot $t+1$, although only community $F$ is the successor of $A$. For realworld networks with hundreds or thousands of communities, the non-representative-based method is very computationally expensive.

\section{Representative-based Method:}

Intuitively, we first find graph representatives in each graph (see Algorithm 1), and we enumerate the communities in each graph using the graph representatives as the seeds to avoid generating redundant communities. We then use community representatives to establish the relationship between the communities from different time stamps (see lines 10-19 in Algorithm 2). If all the predecessors and successors of the community $C_{t}^{i}$ can be found, then we can determine whether $C_{t}^{i}$ is a community dynamic by applying the decision rules (see Section III-B).

For example, in Fig. 3, we use the filled triangle or rectangle nodes as seeds to generate communities. We then look for community representatives (triangular nodes). Taking advantage of the community representatives, we track forward in the sequence to establish the community relationship between the two time stamps. How can we determine if one community at timestamp $t+1$ (or $t$ ) is the successor (or predecessor) of another community at $t$ (or $t+1)$ ? Let us take community $A$ at timestamp $t$, for example. To find the successor of community $A$, first we need to find all communities that contain the community representative of $A$ at timestamp $t+1$. In this case, only community $F$ contains the community representative. Then, we check whether community $F$ meets the condition of successor of $A$ (see Definition 4). Only if this is true, do we establish the relationship between $A$ and $F$. When there are only grown, merged, born or vanished communities like community $A$ grows to community $F$, communities $B$ and $C$ merge into $E$, community $D$ emerges and community $F$ disappears, we do not need to track back in the sequence. In cases of shrunken communities or split communities, we may need to track back. For example, community $D$ shrinks into $I$ with the disappearance of representative 12 at timestamp $t+2$, and community $E$ splits into $H$ and $G$ with representative $3 \in H$ but $3 \notin G$. In this case, we need to use representatives 10 and 6 to track back to establish the relationships between $D$ and $I$, and $E$ and $G$. The workflow of the algorithm is shown in Fig. 4.

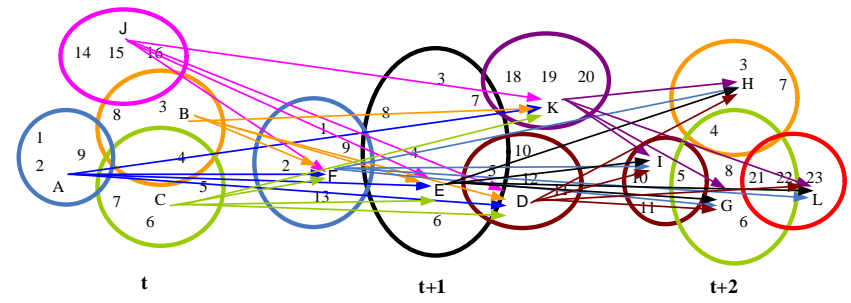

Figure 2. Example for tracking community dynamics using the nonrepresentative-based method. (Best viewed in color.) 


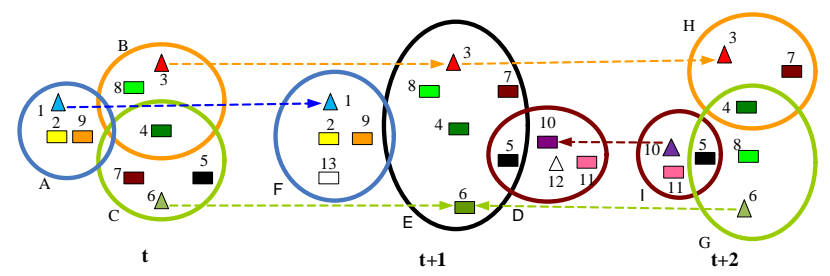

Figure 3. Example for tracking community dynamics using the representative-based method. Note that triangles are community representatives; filled shapes are graph representatives; empty shapes are graphdependent vertices; circles are communities; and dashed lines are community relationships. (Best viewed in color.)

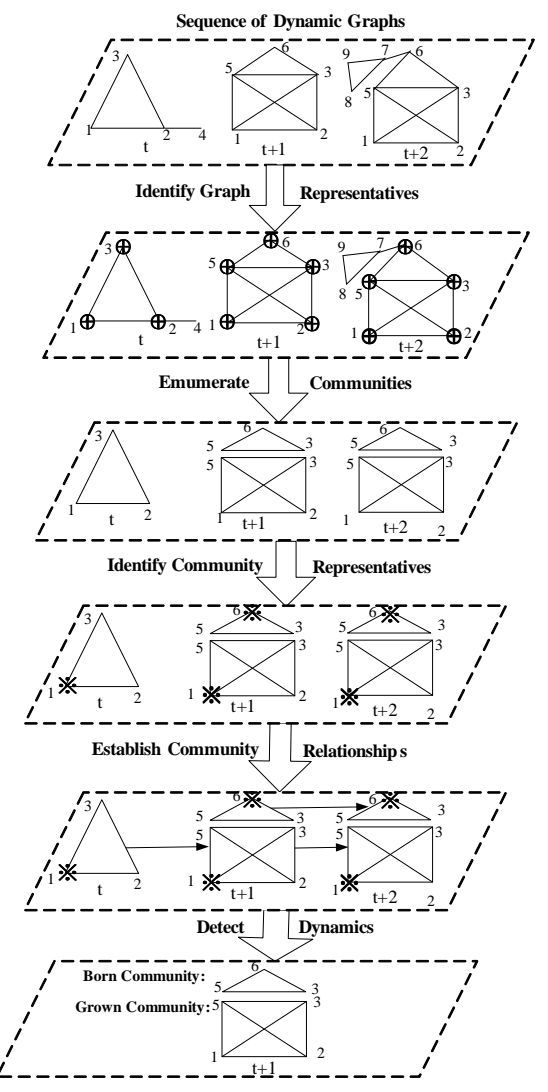

Figure 4. Workflow of the representative-based algorithm with three timestamps.

\section{EXPERIMENTS}

In this section, we evaluate our algorithm on both synthetic and real-world datasets. We focus on answering the following two questions:

1) What is the performance of our algorithm using the representative-based technique?

2) Is our algorithm scalable to large graphs?

3) Does the algorithm provide insights into real-world datasets?

Section IV-A studies the performance of the representative-based algorithm relative to the baseline
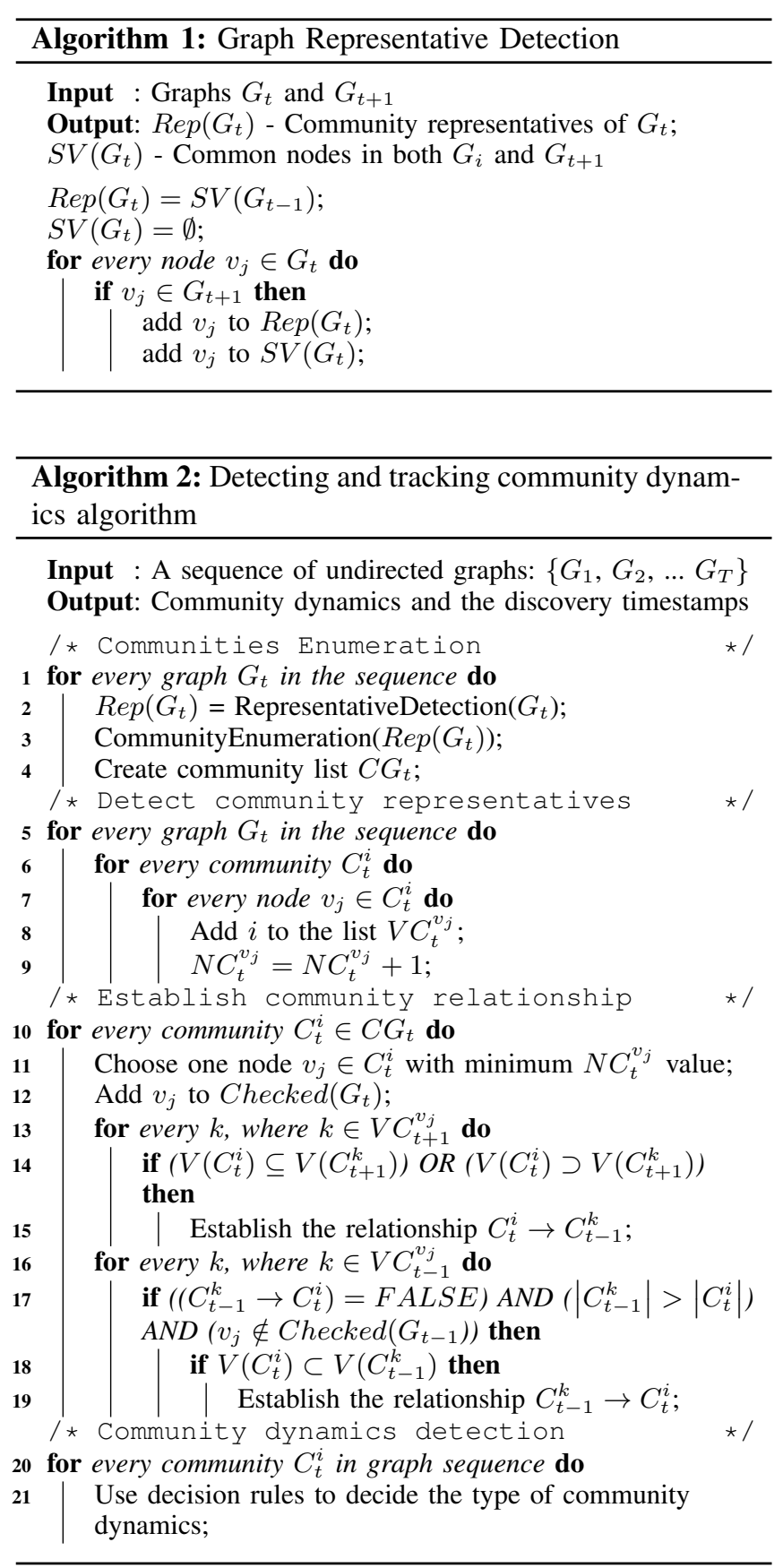

algorithm on synthetic networks. Section IV-B describes our experiments on real networks, including the Food Web and Enron Email datasets. Our experiments were conducted on a PC with an Intel Core 2 Duo CPU $(2.1 \mathrm{GHz})$ and 4GB of RAM. Our algorithm was implemented in the C programming language. We measure the improvement in the runtime of our algorithm versus the non-representativebased algorithm in terms of speedup, which we calculate by dividing the runtime of non-representative-based algorithm into the runtime of our algorithm. 


\section{A. Synthetic Experiments}

In this experiment, we study the effectiveness of the proposed representative-based technique. All the graphs in the synthetic datasets are generated by GTgraph [2] and follow the Recursive Matrix Graph model (R-MAT) [4] so that they have a small-world nature. The parameters for the synthetic graphs, which appear in Table II, are defined as follows: $|V|$ is the number of vertices in a graph, Num $m_{g v}$ is the number of graph-dependent vertices in a graph, and $E_{i}$ is the number of edges in a graph $G_{i}$. On all graphs, we use the default values of $0.45,0.15,0.15$ and 0.25 for the R-MAT parameters a, b, c, d, respectively, with a : b and a : c ratios of 3:1, as in many real world graphs [4]. After graph enumeration, we use a program to relabel some of the vertices according to the parameter $N u m_{g v}$, so that we can have some graph-dependent vertices in each graph when we build the graph sequence. For example, in the dataset syn_500, we can relabel the vertices $v_{j} \in[451,500]$ in graph $G_{i}$ as $v_{j}+50 \times(i-1)$. Other graph-dependent vertices in other datasets can be similarly relabeled.

Table II

TABLE of Synthetic Datasets

\begin{tabular}{|c|c|c|c|c|c|c|c|}
\hline $\begin{array}{l}\vec{D} \\
\tilde{\Xi} \\
\tilde{\tilde{E}} \\
\text { D. }\end{array}$ & $\Sigma$ & 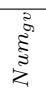 & 国 & 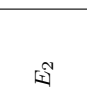 & I & 国 & 迁 \\
\hline syn_500 & 500 & 50 & 8000 & 11000 & 9000 & 12000 & 10000 \\
\hline syn_1000_1 & 1000 & 100 & \multirow{3}{*}{400000} & \multirow{3}{*}{550000} & \multirow{3}{*}{450000} & \multirow{3}{*}{600000} & \multirow{3}{*}{500000} \\
\hline syn_1000_2 & 1000 & 200 & & & & & \\
\hline syn_1000_3 & 1000 & 300 & & & & & \\
\hline syn_1500 & 1500 & 150 & 640000 & 880000 & 720000 & 960000 & 800000 \\
\hline syn_2000 & 2000 & 200 & 800000 & 1100000 & 900000 & 1200000 & 1000000 \\
\hline syn_3000 & 3000 & 300 & 1600000 & 2200000 & 1800000 & 2400000 & 2000000 \\
\hline
\end{tabular}

After building the sequence of graphs, we use the parallel MCE algorithm [14] to enumerate the communities in the individual graphs, and apply the proposed algorithms to detect dynamic changes in the community (or clique) structure between the subsequent graphs. Namely, for the non-representative-based method, we enumerate all the communities in each graph, but for the representativebased algorithm, we use graph representatives as seeds to avoid graph-dependent community enumeration (see a more detailed discussion in Section III-C).

In the first experiment, we try to test the effectiveness of the graph representatives in the community enumeration step. The results appear in Table III: $N C_{n o n}$ is the number of cliques enumerated by the non-representativebased method, $N C_{r e p}$ is the number of cliques enumerated by the representative-based method, $T C_{n o n}$ is the runtime of community enumeration using the non-representativebased method, and $T C_{r e p}$ is the runtime of community enumeration using representative-based method.

As shown in Table III, the representative-based method achieves speedups of more than 1.1 on the dataset syn_1000_1, in which 10 percent of the vertices are graph-
Table III

TABLE OF EFFECTIVENESS OF GRAPH REPRESENTATIVES

\begin{tabular}{|c|c|c|c|c|c|c|}
\hline 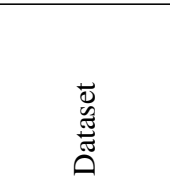 & 这 & 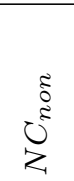 & $\underbrace{\mho^{8}}$ & $\begin{array}{l}\text { की } \\
\text { है } \\
\text { है } \\
\text { हैं } \\
E-1\end{array}$ & $\begin{array}{l}\text { } \\
\text { है } \\
\text { है } \\
\text { के } \\
\text { है }\end{array}$ & 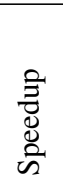 \\
\hline \multirow{5}{*}{ syn_1000_1 } & 400000 & 2505 & 2214 & 9 & 7.9 & 1.14 \\
\hline & 550000 & 2541 & 2299 & 9.4 & 8.4 & 1.12 \\
\hline & 450000 & 2721 & 2336 & 9.8 & 8.4 & 1.17 \\
\hline & 600000 & 2564 & 2303 & 9.3 & 8.3 & 1.12 \\
\hline & 500000 & 2661 & 2341 & 9.7 & 8.4 & 1.15 \\
\hline \multirow{5}{*}{ syn_1000_2 } & 400000 & 2505 & 1773 & 9 & 6.3 & 1.43 \\
\hline & 550000 & 2541 & 1878 & 9.4 & 6.9 & 1.36 \\
\hline & 450000 & 2721 & 1882 & 9.8 & 6.7 & 1.46 \\
\hline & 600000 & 2564 & 1880 & 9.3 & 6.7 & 1.39 \\
\hline & 500000 & 2661 & 1871 & 9.7 & 6.7 & 1.45 \\
\hline \multirow{5}{*}{ syn_1000_3 } & 400000 & 2505 & 1197 & 9 & 4.3 & 2.09 \\
\hline & 550000 & 2541 & 1382 & 9.4 & 5 & 1.83 \\
\hline & 450000 & 2721 & 1158 & 9.8 & 4.1 & 2.39 \\
\hline & 600000 & 2564 & 1221 & 9.3 & 4.4 & 2.11 \\
\hline & 500000 & 2661 & 1278 & 9.7 & 4.6 & 2.11 \\
\hline
\end{tabular}

dependent vertices; speedups of around 1.4 on the dataset syn_1000_2, in which 20 percent of the vertices are graphdependent vertices; and speedups of around 2 on the dataset syn_1000_3, in which 30 percent of the vertices are graph-dependent vertices. The experiments on the datasets syn_500,syn_1500,syn_2000 and syn_3000 also show that the representative-based method can achieve a speedup of at least 1.1 in the community enumeration step, when the dataset has 10 percent graph-dependent vertices.

Table IV

TABLE OF SYNTHETIC EXPERIMENT RESULTS

\begin{tabular}{|c|c|c|c|c|c|c|c|c|}
\hline 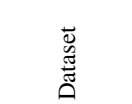 & 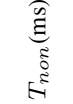 & 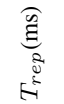 & E & 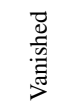 & 䓌 & 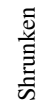 & $\begin{array}{l}\vec{D} \\
\dot{\Phi} \\
\overrightarrow{\tilde{e}}\end{array}$ & $\begin{array}{l}:= \\
\text { के }\end{array}$ \\
\hline syn_500 & 265 & 25 & 3425 & 3482 & 87 & 79 & 18 & 23 \\
\hline syn_1000_1 & 1132 & 87 & 8702 & 8569 & 154 & 119 & 42 & 33 \\
\hline syn_1500 & 6442 & 329 & 23482 & 23621 & 401 & 295 & 71 & 86 \\
\hline syn_2000 & 16182 & 489 & 23111 & 23178 & 333 & 278 & 71 & 83 \\
\hline syn_3000 & 61912 & 1354 & 59261 & 59220 & 813 & 718 & 465 & 313 \\
\hline
\end{tabular}

In the second experiment, we take community representatives into consideration and measure the entire runtime of the two algorithms for detecting the community dynamics across the five timestamps in each of the datasets. After community enumeration, the non-representative-based method would compare all pairs of communities belonging to two consecutive timestamps to build the community relationships, while the representative-based algorithm exploits community representatives to reduce the expensive computational cost of tracking communities. The result of the experiments on the datasets syn_500, syn_1000_1, syn_1500, syn_2000 and 


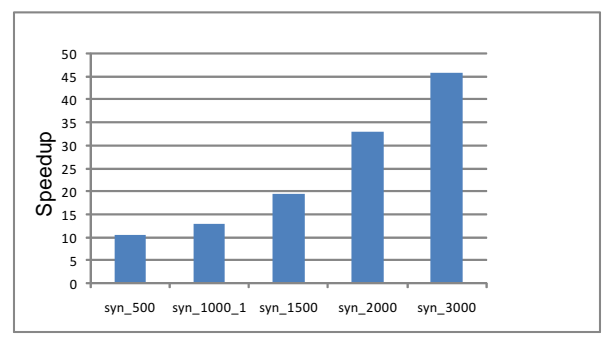

Figure 5. Runtime speedup of the representative-based algorithm over the non-representative-based algorithm for three synthetic datasets.

syn_3000 are shown in Table IV: $T_{\text {non }}$ is the runtime of the non-representative algorithm, $T_{r e p}$ is the runtime of representative algorithm, and the last six columns are the counts of the six types of community dynamics detected by the algorithm. From Fig. 5, we can see that the representativebased algorithm achieves a speedup of 11-46 times with respect to the non-representative-based algorithm.

A time complexity analysis with regard to the number and types of community dynamics would be desirable, but it is hardly possible, because even a single node or edge change in a community may lead to tens of community changes at the same time.

\section{B. Real-world Experiments}

In addition to the synthetic experiments, we applied our algorithm to two real-world dynamic networks including Food Web and Enron Email. The minimum size of communities we considered here is three.

Food Web dataset: The food web consists of marine organisms living in the Chesapeake Bay, containing 33 vertices representing the ecosystem's most prominent taxa, which was originally compiled by Baird and Ulanowicz [3]. Edges between taxa denote trophic relationships-one taxon feeding on another. Here we ignore directionality and consider the network as an undirected graph. Newman [7] has used this dataset as a static graph to detect the communities, while we construct the networks on a seasonal basis from spring to winter to discover community dynamics in dynamic networks.

Table V

TABle of Food Web Communities

\begin{tabular}{|c|c|c|}
\hline Season & Number of Communities & Community Dynamics \\
\hline Spring & 15 & None \\
\hline Summer & 15 & $\begin{array}{l}\text { Four grown communities; One } \\
\text { born community; Four com- } \\
\text { munities will split in fall; } \\
\text { One vanished community; one } \\
\text { merged community }\end{array}$ \\
\hline Fall & 19 & $\begin{array}{l}\text { Two shrunken communities; } \\
\text { four vanished communities } \\
\text { (will disappear in winter) }\end{array}$ \\
\hline Winter & 9 & Four merged communities \\
\hline
\end{tabular}

By applying our algorithm to the Food Web networks, we find all six types of community dynamics (see Table V). Summer is the most active community changing season: four communities grow because microzooplankton, which can not be found in spring, becomes involved in the energy flow network. Four communities split because bacteria do not feed on microzooplankton in fall. The disappearance of sea nettle in fall results in a vanished community (zooplankton, ctenophore, and sea nettle), which was a born community in summer. This indicates that the community is unstable. Due to lack of food in winter, four merged communities emerge in order to get the benefit from more members with the food energy. Typical community dynamic examples discovered in Food Web are shown in Fig. 6-7. We can see that Food Web is characterized by overlapping communities. Note that different cycles represent different communities at the same timestamp.
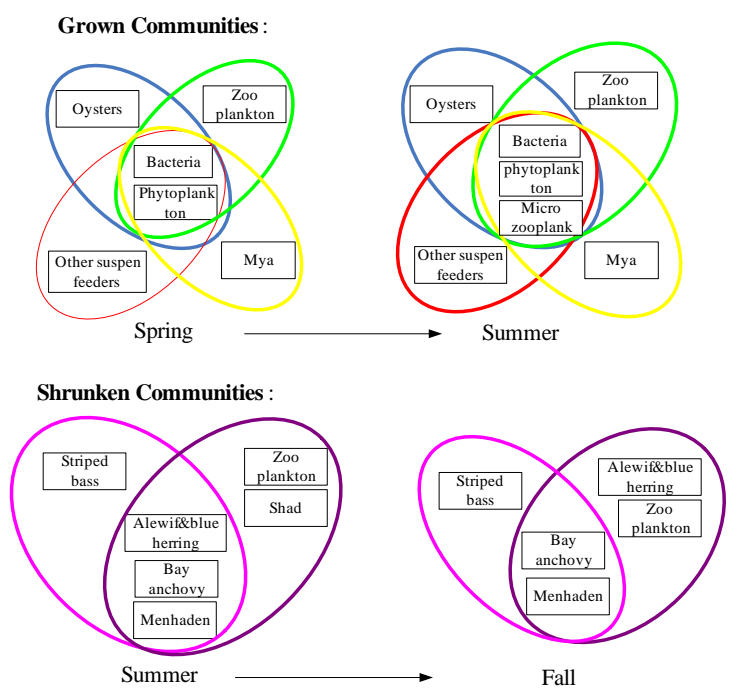

Figure 6. Grown community and shrunken community example of Food Web.
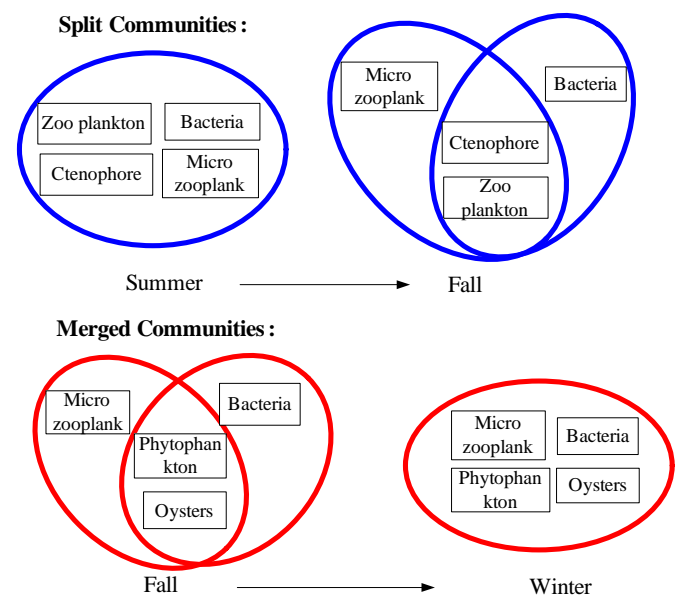

Figure 7. Split community and merged community example of Food Web. 


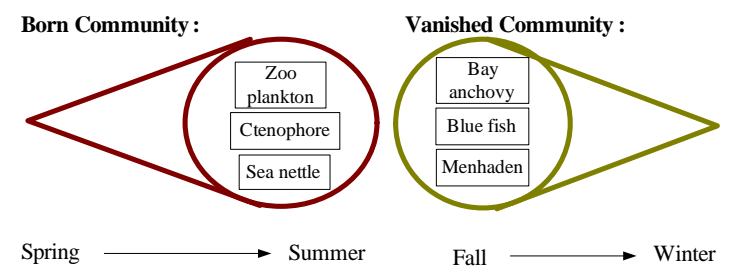

Figure 8. Born community and vanished community example of Food Web.

ENRON dataset: This data set consists of approximately 1.5 million email communications sent or received by employees in Enron, Inc. It is much more complex than the Food Web dataset. We take a subset containing only messages between Enron employees from January to December of 2001 and construct sender-to-recipient undirected graphs on a monthly basis. The graphs have 151 nodes (Enron employees), with low edge density and short average distance between vertices, which shows a "small-world" effect and indicates that the graphs have community structure. The properties of each graph are shown in Table VI.

Table VI

ENRON EMAIL DATASET PROPERTIES

\begin{tabular}{|c|c|c|}
\hline Month & Number of Edges & Number of Communities \\
\hline Jan. & 126 & 21 \\
Feb. & 190 & 56 \\
Mar. & 199 & 54 \\
Apr. & 240 & 66 \\
May & 273 & 90 \\
Jun. & 218 & 49 \\
Jul. & 240 & 68 \\
Aug. & 371 & 120 \\
Sep. & 343 & 110 \\
Oct. & 531 & 196 \\
Nov. & 438 & 143 \\
Dec. & 290 & 93 \\
\hline
\end{tabular}

The community dynamics in each month discovered by our algorithm are shown in Table VII. We can see that there are more community dynamics in October than in any other month. The most likely trigger of this event is the fact that Enron announced a third quarter loss of $\$ 618$ million on October 16 of 2001, which is also thought to be the trigger of the Enron scandal. In order to see the details of the community dynamics in October, let us consider one of the most important nodes-Louise Kitchen, the former President of Enron. There are 20 community dynamics containing Louise Kitchen in October: 16 born communities, 4 grown communities, 1 split community, and 1 shrunken community. From Fig. 9, we can see that some employees like Sally Beck, Chief Operating Officer, joined the senior management communication groups, probably to discuss the serious issues or suggest strategies, while only one personPhillip Allen-left the groups. Confusion among the Enron employees may be why Louise Kitchen's communication groups grew rather than shrank during the turbulent times.

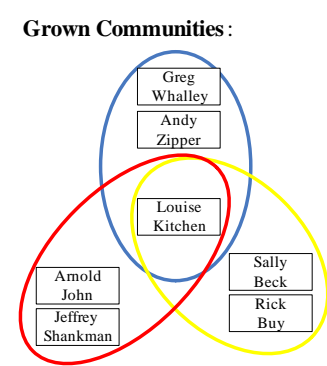

September
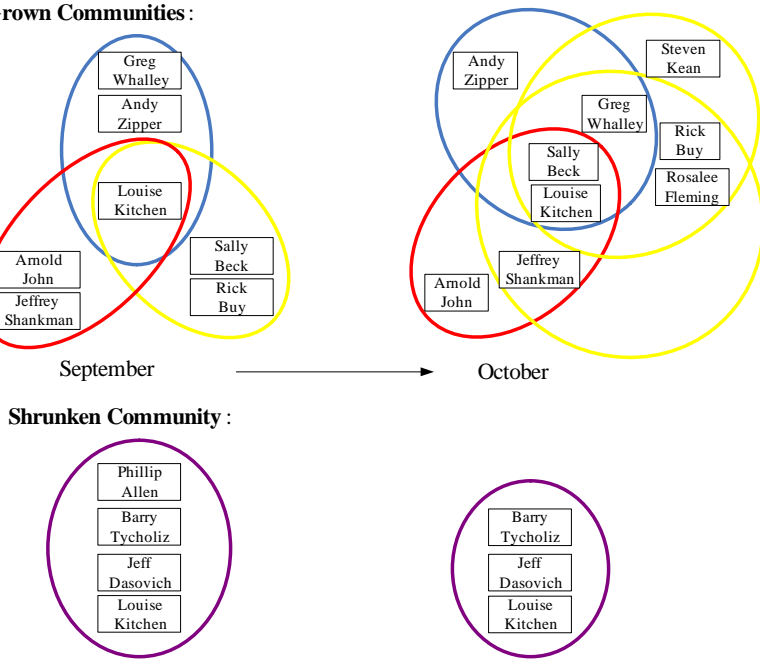

September
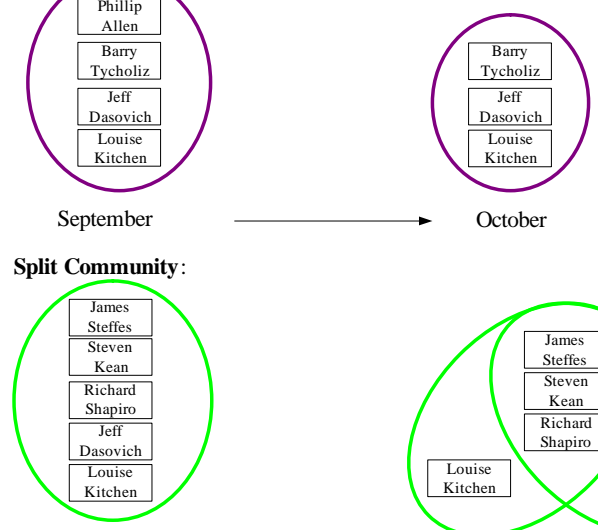

October

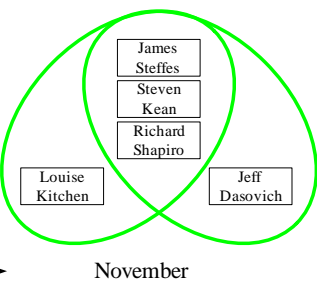

Figure 9. Community dynamics containing Louise Kitchen in October.
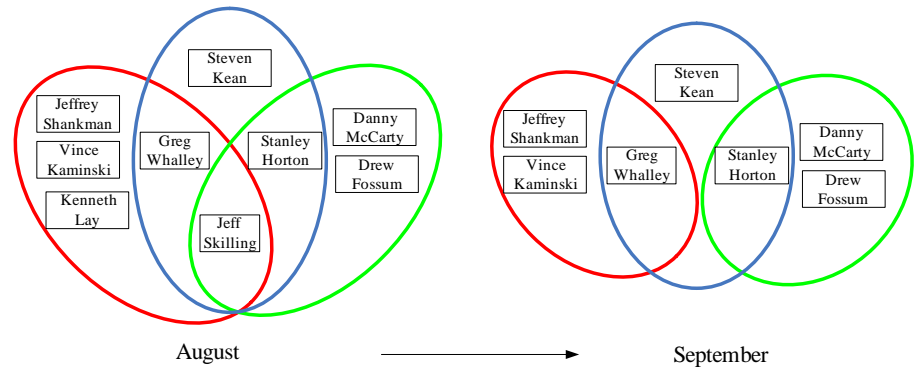

September

Figure 10. Shrunken communities due to Jeff Skillng resigning as CEO in August.

As a second example, take Jeff Skilling, the former CEO of Enron. There are 19 email communities containing Jeff Skilling in August, but among these, 3 communities shrank in September (see Fig. 10), while the other 16 communities disappeared after Jeff Skilling resigned as CEO in August, perhaps because many employees quit or joined other work groups after Skilling's resignation.

\section{CONClusion AND Future Work}

In this paper, we proposed a new method for detecting and tracking all six types of community dynamics, including grown, shrunken, merged, split, born, and vanished communities, in evolutionary networks. Graph representatives 
Table VII

COMMUNITY DYNAMICS IN ENRON EMAIL DATASET

\begin{tabular}{|c|c|c|c|c|c|c|}
\hline Month & Grown & Shrunken & Merged & Split & Born & Vanished \\
\hline Jan. & 0 & 0 & 0 & 0 & 0 & 14 \\
Feb. & 3 & 1 & 0 & 1 & 48 & 32 \\
Mar. & 3 & 2 & 4 & 1 & 33 & 39 \\
Apr. & 6 & 1 & 0 & 2 & 42 & 43 \\
May & 3 & 4 & 0 & 1 & 75 & 76 \\
Jun. & 3 & 3 & 0 & 1 & 34 & 38 \\
Jul. & 1 & 0 & 2 & 0 & 58 & 54 \\
Aug. & 9 & 4 & 2 & 2 & 97 & 89 \\
Sep. & 8 & 9 & 3 & 1 & 79 & 56 \\
Oct. & 30 & 5 & 10 & 8 & 136 & 160 \\
Nov. & 7 & 13 & 0 & 9 & 102 & 97 \\
Dec. & 2 & 17 & 2 & 0 & 54 & 14 \\
\hline
\end{tabular}

and community representatives are defined to reduce the computational cost of the algorithm. The main properties of the method are:

- Parameter-free and automatic;

- Effective and efficient; and

- Applicable to evolutionary networks even characterized with overlapping communities.

We have demonstrated the effectiveness of our method on a number of synthetic examples as well as real-world networks. Experimental results on real-world networks show that our algorithm can detect meaningful community dynamics, and do so in an efficient manner.

Because of lack of benchmark datasets, it is hardly possible to use detection accuracy as one of our metrics. In addition, a time window-based algorithm would be considered in our future work.

\section{ACKNOWLEDGMENT}

This work was funded by the Scientific Data Management Center under the Department of Energy's SciDAC program. The work of ZC was partially supported by the National Science Foundation grant NSF10-564. Oak Ridge National Laboratory is managed by UT-Battelle for the LLC U.S. D.O.E. under contract no. DEAC05-00OR22725.

\section{REFERENCES}

[1] Backstrom, L., Huttenlocher, D., Kleinberg, J., And LAN, X. Group formation in large social networks: membership, growth, and evolution. In Proceedings of the 12th ACM SIGKDD international conference on Knowledge discovery and data mining (2006), ACM, pp. 44-54.

[2] Bader, D. A., And Madduri, K. Gtgraph: A synthetic graph generator suite, 2006.

[3] BAiRd, D., AND Ulanowicz, R. E. The seasonal dynamics of the chesapeake bay ecosystem. Ecological Monographs 59 (1989), 329-364.

[4] Chakrabarti, D., Zhan, Y., And Faloutsos, C. R-mat: A recursive model for graph mining. In In SDM (2004).
[5] Chen, L., DeVries, A. L., And Cheng, C. H. Convergent evolution of antifreeze glycoproteins in Antarctic notothenioid fish and Arctic cod. Proc Natl Acad Sci USA (1997), 38173822 .

[6] Clauset, G., Newman, M. E. And and Moore, C. Finding community structure in very large networks. Physical Review E, (2004), 1-6.

[7] Girvan, M., and Newman, M. E. Community structure in social and biological networks. Proc Natl Acad Sci U S A 99, 12 (June 2002), 7821-7826.

[8] Hendrix, W., Schmidt, M. C., Breimyer, P., And SamATOVA, N. F. On perturbation theory and an algorithm for maximal clique enumeration in uncertain and noisy graphs. In $U$ '09: Proceedings of the 1st ACM SIGKDD Workshop on Knowledge Discovery from Uncertain Data (New York, NY, USA, 2009), ACM, pp. 48-56.

[9] Hopcroft, J., Khan, O., Kulis, B., And Selman, B. Tracking evolving communities in large linked networks. PNAS 101 (2004), 5249-5253.

[10] Kumar, R., Novak, J., And Tomkins, A. Structure and evolution of online social networks. In Proceedings of the 12th ACM SIGKDD international conference on Knowledge discovery and data mining (2006), ACM, p. 617.

[11] Long, M., Betran, E., Thornton, K., And Wang, W. The origin of new genes: glimpses from the young and old. Nat Rev Genet (2003), 865-875.

[12] Palla, G., Derenyi, I., Farkas, I., And Vicsek, T. Uncovering the overlapping community structure of complex networks in nature and society. Nature 435, 7043 (June 2005), 814-818.

[13] Palla, G., lszl Barabsi, A., Vicsek, T., and HunGARY, B. Quantifying social group evolution. Nature 446 (2007), 2007.

[14] Schmidt, M. C., Samatova, N. F., Thomas, K., and PARK, B.-H. A scalable, parallel algorithm for maximal clique enumeration. J. Parallel Distrib. Comput. 69, 4 (2009), $417-$ 428.

[15] Snel, B., Bork, P., And Huynen Genome evolution. Gene fusion versus gene fission. Trends Genet (January 2000), 9-11.

[16] Steinhaeuser, K., Chawla, N. V., and Ganguly, A. R. An exploration of climate data using complex networks. In SensorKDD '09: Proceedings of the Third International Workshop on Knowledge Discovery from Sensor Data (New York, NY, USA, 2009), ACM, pp. 23-31.

[17] Tantipathananandh, C., Wolf, T. B., And Kempe, D. A framework for community identification in dynamic social networks. In KDD '07: Proceedings of the 13th ACM SIGKDD international conference on Knowledge discovery and data mining (2007), ACM, pp. 717-726.

[18] Watts, D. J., And Strogatz, S. H. Collective dynamics of 'small-world' networks. Nature 393, 6684 (1998), 440-442.

[19] Zhang J. Evolution by gene duplication: an update. Trends in Ecology \& Evolution 18 (2003), 292-298. 